\title{
Shock Treatment: Heavy Quark Drag in a Novel AdS Geometry
}

\author{
W. A. Horowitz, ${ }^{*}$ Yuri V. Kovchegov ${ }^{\dagger}$ \\ Department of Physics, The Ohio State University, 191 West Woodruff Avenue, \\ Columbus, OH 43210, USA
}

\begin{abstract}
We calculate the drag force on a heavy quark hit by a shock wave, thus generalizing the strongly coupled AdS/CFT heavy quark drag calculations to both hot and cold nuclear matter. The derivation employs the trailing string configuration, similar to that used in the literature for a quark moving through a thermal medium, though in the shock metric the string profile is described by a much simpler analytic function. Our expression for the drag depends on the typical transverse momentum scale of the matter in the shock. For a thermal medium this scale becomes proportional to the temperature, making our drag coefficient and momentum limit of applicability identical to those found previously. As the shock wave can be composed of either thermalized or non-thermalized media, our derivation extends the existing drag calculations to the case of arbitrarily distributed matter.
\end{abstract}

Keywords: AdS/CFT correspondence, heavy quark energy loss, jet quenching PACS: 11.25.Tq, 12.38.Mh, 24.85.+p, 25.75.-q

\section{Introduction}

The simultaneous emergence of the anti de-Sitter/conformal field theory (AdS/CFT) correspondence $[1-4]$ as a tool to study strongly coupled systems (see $[5,6]$ for reviews) and the failure of perturbative QCD (pQCD) techniques [7] to quantitatively describe the observed heavy ion physics phenomena has inspired both a critical reevaluation of the traditional methods and an explosion of new research [8-14]. In particular a detailed theoretical understanding of high$p_{T}$ particles and jets, coupled with experimental measurements associated with them, holds out the promise of uniquely probing the high- $p_{T}$ physics, low- $p_{T}$ collective physics, and their mutual interaction in a bulk QCD medium. From a theoretical and experimental perspective heavy quark jets offer a rich new set of tools and challenges. Previous AdS/CFT calculations [10-19] of the heavy quark drag assumed a strongly coupled thermal medium of $\mathcal{N}=4$ super Yang-Mills (SYM) plasma. In this Letter we extend the earlier work on the heavy quark drag in a thermal medium to that of an arbitrary medium; in particular our formalism applies to both hot and cold strongly coupled nuclear matter.

The AdS/CFT conjecture postulates a duality between certain field theories and the compactification of Type IIB string theory in various geometries [1-4]. In particular $\mathcal{N}=4 \mathrm{SYM}$

*horowitz@mps.ohio-state.edu

†yuri@mps.ohio-state.edu 
theory is dual to Type IIB string theory in $\mathrm{AdS}_{5} \times \mathrm{S}^{5}$. What makes the conjecture so useful, but also so difficult to prove, is that the weak coupling limit of one theory is dual to the strong coupling limit of the other. Of especial interest to the heavy ion community is the strong coupling limit of QCD, where the only previous theoretical tool was numerical lattice simulations. While lattice simulations remain the only method to obtain quantitative results for strongly-coupled QCD, one may argue that for some observables similarities between QCD and $\mathcal{N}=4 \mathrm{SYM}$ theory can be exploited to improve our qualitative understanding of the former by performing calculations in the latter. In the limit of large 't Hooft coupling and number of colors $N_{c}$, the $\mathcal{N}=4 \mathrm{SYM}$ theory is dual to an (often) analytically tractable theory, the classical limit of string theory: classical supergravity (SUGRA).

Despite the many differences between QCD and $\mathcal{N}=4 \mathrm{SYM}$, there have been a number of qualitative successes in applying the AdS/CFT ideas to heavy ion phenomenology at RHIC. Specifically, the lattice result of a surprising deviation of the entropy density above the deconfinement temperature from the Stefan-Boltzmann limit [20, 21] is easily understood analytically in AdS/CFT [22, 23]; the observed near perfect fluidity of the QGP [24-28] comes from a shear viscosity to entropy ratio an order of magnitude smaller than perturbative estimates, but in line with the AdS/CFT calculations [8]; the shockingly large suppression of heavy quarks [29-33] appears to be easier to describe in the AdS/CFT framework [10-13, 15-18]; and the emergence of an away-side double hump structure in two [34-36] and three particle [37, 38] correlators, while suggestive of a Mach cone formed by a supersonic jet [39, 40], can currently only be understood as the result of the near field energy and momentum deposition derived using AdS/CFT $[19,41,42]$.

On the other hand, from both experimental and theoretical standpoints, perturbative methods as applied to jet quenching physics should be viewed with increased skepticism. Early quantitative success in describing the energy loss of light partons [43-45] as evidenced by the suppression of pions [46, 47], etas [48], and the null control of direct gammas [49, 50] has given way to an inability $[7,51,52]$ to simultaneously describe even any two of the following four observables: the (1) pion quenching and (2) similarly large suppression of non-photonic electrons (NPE), the decay fragments of heavy quarks, and the surprisingly large azimuthal anisotropy of the (3) hadrons from light parton jets [53, 54] and of the (4) NPE [31]. From a self-inconsistency standpoint the assumption of a small coupling, $g \ll 1$, in high- $p_{T}$ energy loss has never been true - at RHIC energies and temperatures $g \sim 2$-with the momentum scale, set by either the Gyulassy-Wang model [55] of Yukawa-like scattering centers or the saturation scale, on the order of $0.5-1 \mathrm{GeV} \sim \mathcal{O}(1) \Lambda_{Q C D}$, far from perturbative.

An understanding of the limits of applicability, of the inherent theoretical error, are required in order to either gain confidence in or falsify a calculation with experimental data. In deep inelastic scattering (DIS) and Drell-Yan production, the rigorous framework of factorization allows for a well-controlled pQCD expansion (see [56] and references therein). For heavy ion collisions it is not clear that factorization holds any longer [57]. Even supposing it does, the numerical value of the momentum characterizing the scale at which pQCD methods break down is not quantitatively known. The AdS calculations are under even less control. Until a dual string geometry is found for QCD one cannot quantitatively estimate the changes in observables from using a different theory; there is no path through dual theories approaching QCD whose parameterization may be used as an expansion parameter.

What one can do is explore the results from different theories and geometries in an attempt 
to discover universal behavior. This may give one reason to believe that the result will hold for QCD, at least at the qualitative level. In this Letter we follow this strategy by considering the heavy quark momentum loss in a novel geometry. Previous drag force calculations focused on motion of the gauge theory quark in vacuum (empty AdS metric) [15, 17, 18], or in a static thermal medium (black hole and black hole-like metrics) [10-13, 16, 17]. Here we examine the drag force on a static quark inside a shock wave. In particular the shock wave can be a model of the dense medium produced in a heavy ion collision. Equivalently it can also be a model of a nucleus incident on a probe quark, giving rise to cold-matter energy loss as is often considered in proton-nucleus collisions.

The paper is structured as follows. In Sect. 2 we present the shock wave metric and solve the string equations of motion to find the trailing string solution, similar to [11-13]. We note that the analytic form of the trailing string solution, given below in Eq. (13), is considerably simpler than that found in [11-13]. In Sect. 3 we use the solution from Eq. (13) obtained in Sect. 2 to calculate the drag force on the quark inside the shock wave. Boosting the resulting expression to the rest frame of the medium and taking its typical momentum to be set by the temperature we obtain the same equation for the drag force as derived in the original works [11-13]. Just as the form of perturbative jet energy loss is independent of the state of the medium, it turns out that the heavy quark energy loss in a shock wave is exactly the same as that in a thermalized medium. Our result is derived for a shock wave made of any distribution of matter: due to Lorentz time-dilation the shock wave may represent a "snapshot" of any non-equilibrium matter. Hence our result generalizes the drag force of [11-13] to the case of a non-thermal medium. We also study the limitations of our calculation in Sect. 4 and find that the momentum "speed limit" of applicability for the shock wave calculation is parametrically exactly the same as for the black hole metric. We conclude in Sect. 5 by restating our main results and discussing the relation between the quark mass in vacuum and in medium for strongly-coupled gauge theories.

\section{Shock Metric, EOM, and the Trailing String Solution}

We will consider the generalized "shock" metric [9]

$$
\begin{aligned}
d s^{2} & \equiv G_{\mu \nu} d x^{\mu} d x^{\nu}=\frac{L^{2}}{z^{2}}\left[-2 d x^{+} d x^{-}+2 \mu z^{4} \theta\left(x^{-}\right) d x^{-2}+d x_{\perp}^{2}+d z^{2}\right] \\
& =\frac{L^{2}}{z^{2}}\left[-\left(1-\mu z^{4} \theta\left(x^{-}\right)\right) d t^{2}-2 \mu z^{4} \theta\left(x^{-}\right) d t d x+\left(1+\mu z^{4} \theta\left(x^{-}\right)\right) d x^{2}+d x_{\perp}^{2}+d z^{2}\right]
\end{aligned}
$$

where we have used the $x^{ \pm}=(t \pm x) / \sqrt{2}$ normalization of light-cone coordinates with $x=x^{3}$ and dropped the $d \Omega_{5}^{2}$ standard metric of the five-sphere in $\operatorname{AdS}_{5} \times S^{5}$. As usual $L$ is the radius of the $S^{5}$ space. Also $d x_{\perp}^{2}=\left(d x^{1}\right)^{2}+\left(d x^{2}\right)^{2}$ is the transverse part of the metric, and $\mu, \nu=0, \ldots, 4$.

The physical justification for describing Eq. (1a) as a shock metric comes from the application of holographic renormalization [58], which relates the metric in Fefferman-Graham form [59] in $d+1$ dimensions to the energy-momentum tensor in the $d$ dimensional boundary theory. 
For our particular choice of metric, Eq. (1a), the above prescription yields

$$
\left\langle T_{--}\right\rangle=\frac{N_{c}^{2}}{\pi^{2}} \mu \theta\left(x^{-}\right)
$$

which describes an ultrarelativistic shock wave (shock front) moving in the positive $x^{3}=x$ direction.

Previous calculations used $\mu \delta\left(x^{-}\right)$as the coefficient of the $d x^{-2}$ term in Eq. (1a) to represent the Lorentz-contracted pancake of nuclear matter probed in DIS [60] or seen in heavy ion collisions [61]. Note that in those papers $\mu$ is a slightly different quantity with units $\mathrm{GeV}^{3}$, unlike $\mathrm{GeV}^{4}$ here. As observed in [9] the coefficient of $d x^{-2}$ can be any function of $x^{-}$; the resulting metric still satisfies Einstein's equations. We take it $\propto \theta\left(x^{-}\right)$to represent an incoming dense medium of nuclear matter colliding with a heavy quark in its rest frame. The setup is shown in Fig. 1, where the shaded region is the incoming shock wave. The fifth dimension $z$ increases as one moves down the $z$ axis: the top of the diagram is the boundary of $\operatorname{AdS}_{5}$ space $(z=0)$; at the bottom $(z=\infty)$ is the stack of $N_{c}$ D3 color branes. A finite mass fundamental representation heavy quark in the 4D field theory corresponds in the 10 dimensional supergravity theory to the endpoint of an open Nambu-Goto string terminating on a D7 brane; the other end of the open string ends on the $N_{c}$ stack of D3 branes at $z=\infty$. The D7 brane wraps an $S^{3} \subset S^{5}$ and fills the asymptotically AdS space from $z=0$ down to $z=z_{M}$ [62]. Before the collision the string hangs straight down (left panel of Fig. 1), while after the collision the string trails behind the quark (right panel of Fig. 1).

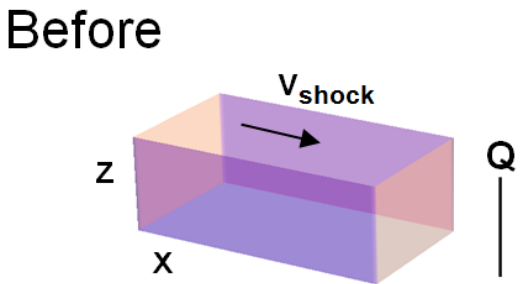

(a)
After

\section{(b)}

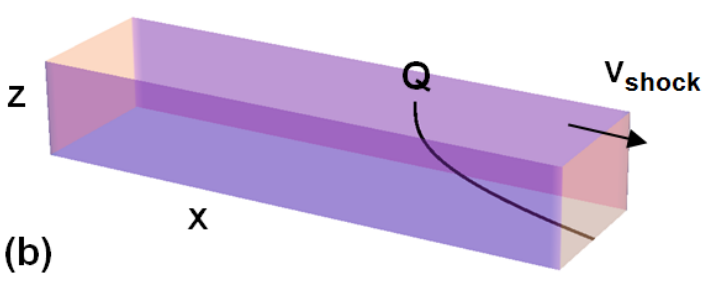

Figure 1: Cartoon of the shock medium colliding with the heavy quark $Q$ in its rest frame. (a) Before the collision the string representing the heavy quark hangs from the D7 brane straight down to the D3 branes at the origin. (b) After the collision the string trails behind $Q$. As the shock is moving in the positive direction $\left(v_{\mathrm{sh}}>0\right)$ in the heavy quark rest frame momentum actually flows up the string as the shock tries to transfer positive momentum to the quark; see Sect. 3 for more details.

Ordinarily the spatial direction picked out when using light cone coordinates represents the beam direction. That would be the case here if we apply our model to the description of energy loss in proton-nucleus collisions, i.e., in cold nuclear matter. For jet energy loss in heavy ion collisions $x$ corresponds to the direction of motion of the heavy quark in the lab frame, an orientation often taken transverse to the beam.

While [11] extended the heavy quark drag calculations to more general metrics of the black hole type, a novel feature of the $G_{\mu \nu}$ used here is its lack of an event horizon. If light can pass through a surface coming from inside the suspected black hole, the surface is not a true horizon. For our metric a light ray moving towards the boundary of AdS space both in the $z$ 
and $x$ direction can cross the suspected horizon at $z_{h}=\mu^{-1 / 4}$. Since the light ray can therefore escape from inside the suspected black hole, the surface at $z_{h}=\mu^{-1 / 4}$ is not a true horizon.

We are interested in the motion of a heavy quark in the background specified by the metric of Eq. (1b). The test string action is

$$
\begin{aligned}
S_{N G} & =-T_{0} \int d \tau d \sigma \sqrt{-g} \\
g & =\operatorname{det} g_{a b}, \quad g_{a b}=G_{\mu \nu} \partial_{a} X^{\mu} \partial_{b} X^{\nu},
\end{aligned}
$$

where $G_{\mu \nu}$ is the spacetime metric of Eq. (1b), Greek indices refer to spacetime coordinates, and Latin indices to worldsheet coordinates. $X^{\mu}=X^{\mu}(\sigma)$ specifies the mapping from the string worldsheet coordinates $\sigma^{a}$ to spacetime coordinates $x^{\mu}$. The backreaction of the fundamental string $\left(\mathcal{O}\left(N_{c}\right)\right)$ is neglected as compared to the $\mathcal{O}\left(N_{c}^{2}\right)$ contributions from the adjoint fields of $\mathcal{N}=4$ SYM [63].

Varying the action, Eq. (3), yields the equations of motion:

$$
\nabla_{a} P_{\mu}^{a}=0, \quad P_{\mu}^{a}=\pi_{\mu}^{a} / \sqrt{-g}=-T_{0} G_{\mu \nu} \partial^{a} X^{\nu},
$$

where $\nabla_{a}$ is the covariant derivative with respect to the induced metric, $g_{a b}$, and the $\pi_{\mu}^{a}$ are the canonical momenta,

$$
\pi_{\mu}^{a}=-T_{0} \frac{\partial \sqrt{-g}}{\partial\left(\partial_{a} X^{\mu}\right)} .
$$

We limit our attention to only the nontrivial directions of $t, z$, and $x$, where $x$ is parallel to the direction of the heavy quark propagation in the lab frame (although we will carry out our derivations in the rest frame of the heavy quark). $X^{\mu}(\sigma)$ maps into the $(t, x, z)$ coordinates; choosing the static gauge, $\sigma^{a}=(t, z)$, the string embedding is described by a single function, $x(t, z)$ :

$$
X^{\mu}(\sigma)=(t, 0,0, x(t, z), z) .
$$

The equations of motion for $x(t, z)$ can be derived in two equivalent ways: plug in the $x(t, z)$ ansatz into Eq. (4); or first substitute the ansatz into Eq. (3), then vary the action. Following the latter approach and denoting $\partial_{t} x=\dot{x}$ and $\partial_{z} x=x^{\prime}$ we find

$$
\begin{gathered}
-g=\frac{L^{4}}{z^{4}}\left(1+x^{2}-\dot{x}^{2}-\mu z^{4}(1-\dot{x})^{2}\right), \\
\frac{\partial}{\partial t}\left(\frac{\mu z^{4}-\left(1+\mu z^{4}\right) \dot{x}}{z^{4} \sqrt{-g}}\right)+\frac{\partial}{\partial z}\left(\frac{x^{\prime}}{z^{4} \sqrt{-g}}\right)=0 .
\end{gathered}
$$

One may determine the mass of the equivalent point particle in the $4 \mathrm{D}$ field theory by examining the string motion outside the shock. For $\mu=0$, the constant velocity string $x(t, z)=$ $x_{0}+v t$ is clearly a solution to the equations of motion, Eq. (8); the action, Eq. (3), becomes

$$
S=-T_{0} L^{2} \int_{z_{M}}^{\infty} d z \frac{1}{z^{2}} \int d t \sqrt{1-v^{2}},
$$

the action of a point particle of mass $M_{q}=T_{0} L^{2} / z_{M}$. Hence we have that

$$
z_{M}=\frac{T_{0} L^{2}}{M_{q}}=\frac{\sqrt{\lambda}}{2 \pi M_{q}},
$$




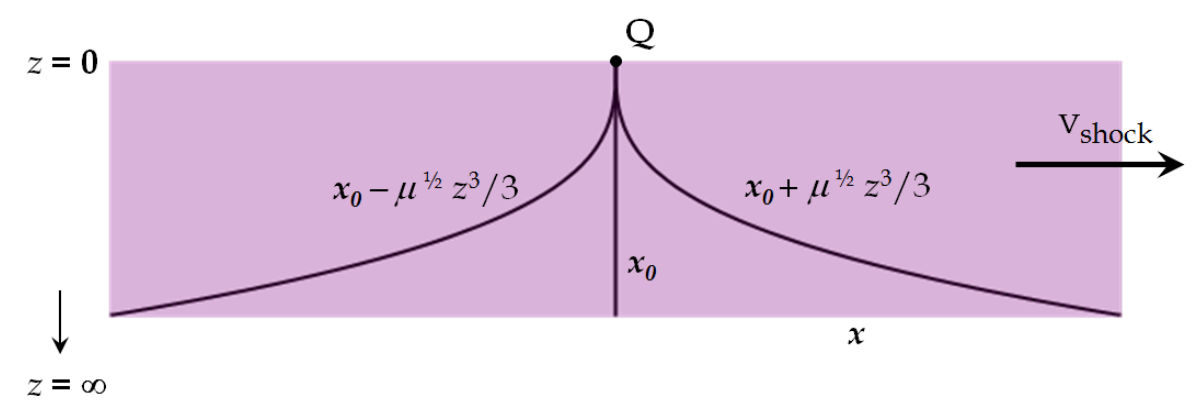

Figure 2: The three solutions, $x(t, z)=\xi(z)=x_{0}, x_{0} \pm \sqrt{\mu} z^{3} / 3$, to the equation of motion for a static string inside the shock, Eq. (11). The constant solution $\left(x(t, z)=x_{0}\right)$ is unstable, $S \sim i \infty$. We also throw away the unphysical time-reversed solution $\left(x(t, z)=x_{0}-\sqrt{\mu} z^{3} / 3\right)$ by noting that any fluctuation would cause it to move toward the remaining (physical) one (see the text for details).

where we have used the AdS dictionary to relate $T_{0} L^{2}=\sqrt{\lambda} / 2 \pi$.

Assuming an asymptotic static solution, $x(t, z)=\xi(z)$, in a metric for which the shock occupies all space and time, the induced metric becomes time-independent. The equations of motion, Eq. (8), reduce to

$$
\frac{\partial}{\partial z}\left(\frac{\xi^{\prime}}{z^{4} \sqrt{-g}}\right)=0
$$

Consider the first integral of the motion resulting from Eq. (11). If we set the quantity in parentheses in Eq. (11) equal to a constant $C$, then we can solve for $\xi^{\prime}$ :

$$
\xi^{\prime}(z)= \pm C z^{2} \sqrt{\frac{1-\mu z^{4}}{1-C^{2} z^{4}}} .
$$

There are two cases to consider for a string hanging from the boundary down to the stack of D3 branes: $C=0$ and $C \neq 0$. (For other solutions of the equations of motion see Appendix A.) For $C \neq 0$, the constant of integration is fixed by considering the signs of the numerator and denominator inside the radical as a function of $z$ : for small $z$ both are positive; for large $z$ both are negative. In order to avoid imaginary solutions, the numerator and denominator must change signs at precisely the same value of $z$; thus $C=\sqrt{\mu}$. This leads immediately to

$$
\xi(z)=x_{0} \pm \frac{\sqrt{\mu}}{3} z^{3},
$$

which are shown in Fig. 2. It is amusing to note that the near-boundary expansion of the static quark solution for the black hole metric (with horizon at $z=z_{h}$ ) is $x(t, z) \approx x_{0}+v t \pm z^{3} /\left(3 z_{h}^{2}\right)$. However we would like to point out that the string configuration in Eq. (13) is valid for all $z$ in the shock metric and is thus significantly simpler than that found in the black hole metric. As we will see below, while being analytically simpler, our solution retains all the main qualitative features of the black hole case.

The sign ambiguity, resulting from the time-reversal symmetry of the problem, can be fixed by taking the positive, trailing solution; the negative sign has the string "trailing" in front of the heavy quark. We find from Sect. 3 that, in the lab frame, the physical solution loses 
momentum to the medium whereas the unphysical solution gains it. Additionally we will find in Sect. 4 that any fluctuation of the negative sign string solution will lead to it approaching its time-reversed, physical solution.

Since the heavy quark is static inserting Eq. (13) back into the action and integrating out the $z$ coordinate yields a point particle action $S=-M_{q} \int d t$ with the vacuum heavy quark mass $M_{q}$. This should be contrasted to the result found in the black hole metric for which the point particle mass is modified from that in vacuum - interestingly, it is decreased in medium. We will discuss this further in the conclusions, Sect. 5.

For $C=0, \xi=x_{0}$ is immediately found: the string hangs straight down. However plugging the solution back into the action gives

$$
S=-T_{0} \int d t \int_{z_{M}}^{\infty} d z \frac{1}{z^{2}} \sqrt{1-\mu z^{4}}
$$

One sees that the IR (large- $z$ ) part of the $z$ integration gives the action an infinite imaginary part. We interpret this as an infinitely unstable state that would immediately decay into the physical trailing string solution. This and the two solutions from Eq. (13) are shown in Fig. 2.

\section{Drag Force}

The drag force on the heavy quark in the SYM theory corresponds to the momentum flow from the direction of heavy quark propagation down the string, i.e., $d p / d t=-\pi_{x}^{1} . \pi_{\mu}^{a}$ are the canonical momenta and may be found from Eqs. (5) and (7):

$$
\begin{aligned}
& \left(\begin{array}{c}
\pi_{t}^{0} \\
\pi_{x}^{0} \\
\pi_{z}^{0}
\end{array}\right)=\frac{T_{0} L^{4}}{z^{4} \sqrt{-g}}\left(\begin{array}{c}
-1-x^{2}+\mu z^{4}(1-\dot{x}) \\
\dot{x}-\mu z^{4}(1-\dot{x}) \\
-x^{\prime}\left(\dot{x}-\mu z^{4}(1-\dot{x})\right)
\end{array}\right) \\
& \left(\begin{array}{c}
\pi_{t}^{1} \\
\pi_{x}^{1} \\
\pi_{z}^{1}
\end{array}\right)=\frac{T_{0} L^{4}}{z^{4} \sqrt{-g}}\left(\begin{array}{c}
\dot{x} x^{\prime} \\
-x^{\prime} \\
-1+\dot{x}^{2}+\mu z^{4}(1-\dot{x})^{2}
\end{array}\right)
\end{aligned}
$$

The "momentum change" of our heavy quark solution given by Eq. (13), where momentum change is in quotation marks as the quark is held static, is then

$$
\frac{d p}{d t}=-\pi_{x}^{1}=\frac{\sqrt{\lambda}}{2 \pi} \sqrt{\mu}
$$

Since all our calculations have been in the heavy quark rest frame, Eq. (17) does not describe the momentum loss for a heavy quark in the lab frame, the rest frame of the shock. Even though formally the shock propagates at the speed of light we will think of it as an approximation to the physical setup of a medium moving almost on the light-cone; then this rest frame is well defined. Moreover we can now also relate $\mu$ to properties of the medium by comparing the energy-momentum tensor found from the metric using the holographic renormalization procedure, Eq. (2), to one derived from the medium properties.

Following [60], we assume the medium is made up of $N_{c}^{2}$ valence gluons of the $\mathcal{N}=4 \mathrm{SYM}$ fields; see Fig. 3. If in the rest frame of the medium the particles are isotropically distributed 
with a typical momentum of order $\Lambda$ - with associated inter-particle spacing of order $1 / \Lambda$ - then the 00 component of the stress-energy tensor in the rest frame of the shock is

$$
\left\langle T_{00}^{\prime}\right\rangle \propto N_{c}^{2} \Lambda^{4}
$$

where primes denote quantities in the rest frame of the medium and proportionality is up to a constant numerical factor. Changing into lightcone coordinates and boosting into the rest frame of the heavy quark yields

$$
\left\langle T_{--}\right\rangle=N_{c}^{2} \Lambda^{4} \gamma^{2}=N_{c}^{2} \Lambda^{4}\left(\frac{p^{\prime}}{M}\right)^{2},
$$

where we assumed ultrarelativistic motion for the heavy quark in the medium rest frame, $p^{\prime} \simeq M \gamma$. (Eq. (19) can be viewed as a definition of the scale $\Lambda$ absorbing numerical constants arising from the precise definition of $\left\langle T_{00}^{\prime}\right\rangle$ and from the change to $\left\langle T_{--}\right\rangle$.) Comparing this with Eq. (2) we read off

$$
\mu=\pi^{2} \Lambda^{4}\left(\frac{p^{\prime}}{M}\right)^{2} .
$$

We now have the right hand side of Eq. (17) in terms of the momentum of the heavy quark in the rest frame of the medium; however the left hand side is still evaluated in the heavy quark rest frame.
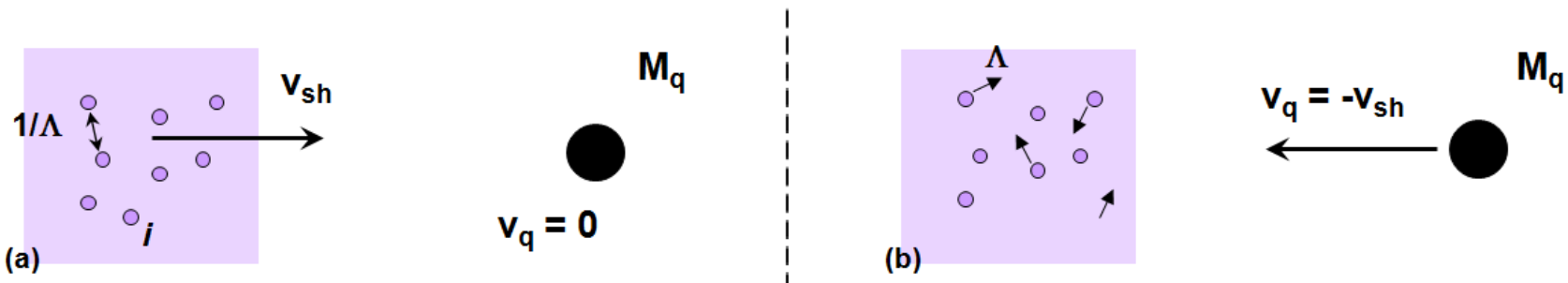

Figure 3: Illustration of the shock wave colliding with a heavy quark in the (a) heavy quark rest frame and (b) medium rest frame. In (a) the shock moves with velocity $v_{s h}$ in the positive $x$ direction; in (b) the heavy quark has momentum $p^{\prime} \simeq \gamma M_{q}$ in the negative $x$ direction.

To rewrite $d p / d t$ on the left of Eq. (17) in terms of the momentum and time in the medium rest frame, $d p^{\prime} / d t^{\prime}$, note that $d p / d t$ is the 3 -vector component of the force 4 -vector in the quark rest frame:

$$
f^{x} \equiv \frac{d p}{d \tau}=\frac{d p}{d t}
$$

One can see from Eq. (16) that $\pi_{t}^{1}=0$, and hence $f^{t}=0$, and the 4 -force boosted into the shock rest frame is therefore

$$
f^{\prime x}=-\gamma f^{x}=-\gamma \frac{d p}{d t},
$$

where the negative sign comes from boosting into a frame moving in the opposite direction; see Fig. 3. From the definition of the 4-force we also know that in this frame

$$
f^{\prime x} \equiv \frac{d p^{\prime}}{d \tau}=\gamma \frac{d p^{\prime}}{d t^{\prime}}
$$


Hence we find that

$$
\frac{d p}{d t}=-\frac{d p^{\prime}}{d t^{\prime}}
$$

Eqs. (17), (20), and (24) lead us to the main result of this Letter:

$$
\frac{d p^{\prime}}{d t^{\prime}}=-\frac{\sqrt{\lambda}}{2} \frac{\Lambda^{2}}{M_{q}} p^{\prime}
$$

Should we take the typical medium particle momentum to be $\Lambda=\sqrt{\pi} T$, reminiscent of the thermal equilibrium result, then our result exactly reproduces that of the black hole metric, $d p^{\prime} / d t^{\prime}=-\pi \sqrt{\lambda} T^{2} p^{\prime} /\left(2 M_{q}\right)[11-13]$.

\section{Limits of the Calculation}

As shown in $[12,13,63]$ there are limits to the applicability of the heavy quark drag calculations in a black hole metric. In the original analytic construction the heavy quark was taken to have constant velocity, despite the momentum lost to the medium. If one proposes the existence of a field on the D7 brane supporting the finite mass heavy quark motion, then one is naturally led to a speed limit based on the maximum field strength supported by the Born-Infeld action. However numerical calculations were made for quarks with non-constant velocity and it turned out that the drag form derived analytically is a very good approximation for this more difficult problem. Nevertheless one might still have a speed limit induced by the warping of spacetime due to the presence of the thermal black hole. The finite heavy quark mass means that the string endpoint representing the quark in $4 \mathrm{D}$ propagates at $z=z_{M}$ in the $5^{\text {th }}$ dimension (see Eq. (10)). While at the Minkowski boundary, $z=0$, the flatness of spacetime gives a local speed of light $c=1$, reality of the point particle action in 5 bulk dimensions in the AdS BH metric with the horizon at $z=z_{h}$ requires that $\sqrt{-G_{\mu \nu}\left(d x^{\mu} / d \tau\right)\left(d x^{\nu} / d \tau\right)}=\sqrt{\left(1-\left(z / z_{h}\right)^{4}\right) / z^{2}-v^{2} / z^{2}}$ be real. Then $v^{2}<1-\left(z / z_{h}\right)^{4}$ leading to $\gamma<\left(z_{h} / z\right)^{2}<\left(z_{h} / z_{M}\right)^{2}[12,13,63]$. The speed limits resulting from these two lines of reasoning are precisely the same; this is not an accident but due to T-duality [64].

While the metric, Eq. (1b), does not support an event horizon, reality of the point particle action still yields an asymmetric, z-dependent result for the local speed of light; to wit, in the rest frame of the heavy quark,

$$
\frac{\mu z^{4}-1}{\mu z^{4}+1} \leq v \leq 1 .
$$

At the boundary, $z=0$, the usual Minkowski speed of light limit, $-1 \leq v \leq 1$, is recovered. Motion at the stack of D3 color branes (at $z=\infty$ ) is restricted to the speed of light in the direction of the shock medium motion.

In a similar way one finds that reality of the Nambu-Goto action requires the velocity of the string at $z=\infty$ to be +1 . This provides an argument against the reversed trailing string solution (given by Eq. (13) with the minus sign on its right hand side): the only classical fluctuations with non-zero velocity supported by it are those that, at the D3 branes at $z=\infty$, give motion at the speed of light in the direction of the shock motion, i.e., towards the physical trailing string solution (given by Eq. (13) with the plus sign on the right hand side). This 
demonstrates that the reversed trailing string solution is unstable under a large set of string fluctuations, and can therefore be discarded. (This conclusion should be compared to the stability analysis carried out in [65] for the black hole metric.)

Plugging $v=0$ for our static quark into Eq. (26) gives us the bound for this calculation, namely

$$
\mu z_{M}^{4} \leq 1
$$

Using Eq. (10) to write $z_{M}=\sqrt{\lambda} /\left(2 \pi M_{q}\right)$ and Eq. (20) for $\mu=\pi^{2} \Lambda^{4} \gamma^{2}$, along with $\Lambda=\sqrt{\pi} T$, we obtain

$$
\gamma \leq \frac{4 \pi M_{q}^{2}}{\lambda \Lambda^{2}}=\frac{4 M_{q}^{2}}{\lambda T^{2}}
$$

The speed limit in this geometry is therefore identical to that for the BH metric $[13,63,65]$.

\section{Conclusions and Discussions}

In this Letter we used the AdS/CFT correspondence to calculate the drag force on a heavy quark in a strongly coupled medium. By placing the string in a shock metric, we extended the derivation of drag in a thermalized medium - whose metric contains a black hole - to a generalized medium not necessarily thermalized. It turns out that, just as for perturbative energy loss, the form of the drag is independent of whether the medium is thermal or not. Moreover the momentum speed limit for the applicability of our calculations in the shock metric is the same as was found for the black hole metric.

To complete the comparison of our calculation with the existing ones for the drag force in a thermal medium $[12,13,63]$ let us find the mass of the heavy quark in the shock wave. According to the standard prescription $[12,13,63]$ the (static) quark mass in the shock is

$$
M_{q}^{\text {shock }}=-\int_{z_{M}}^{\infty} d z \pi_{t}^{0}
$$

which, with the help of Eq. (15) and Eq. (13) yields

$$
M_{q}^{\text {shock }}=\frac{\sqrt{\lambda}}{2 \pi} \int_{z_{M}}^{\infty} \frac{d z}{z^{2}}=\frac{\sqrt{\lambda}}{2 \pi} \frac{1}{z_{M}}=M_{q} .
$$

We see that the quark mass does not change inside the shock wave as compared to the mass in the empty space outside of the shock wave. This is in stark contrast to the thermal modification of the quark mass observed in [63] for a quark in a finite-temperature medium.

To understand this result let us first discuss the origin of the medium modification of the quark mass found in [63]. ${ }^{\ddagger}$ In [63] it was found that at finite temperature $T$ the quark mass

\footnotetext{
${ }^{\ddagger}$ We thank Cyrille Marquet for a discussion which led us to the qualitative understanding of the medium modification mechanism described below.
} 
becomes $M_{q}(T)=(\sqrt{\lambda} / 2 \pi)\left[\left(1 / z_{M}\right)-\left(1 / z_{h}\right)\right]$ with the horizon radius $z_{h}=1 / \pi T$. The mass of the heavy quark in AdS/CFT is constructed by integration over the length of the string, as shown in Eq. (29). On the gauge theory side this implies that the mass is built up from radiative corrections: at strong coupling the corrections are large and lead to a large dressed quark mass [66]. That is why the quarks in AdS/CFT are always heavy. Thinking of a heavy quark as being dressed by a cloud of strongly coupled particles ("partons") it is easy to understand the field theoretic origin of medium modification of the quark's mass, due on the AdS side to the $z_{h}$ IR cutoff in the $z$-integration introduced by the $\mathrm{BH}$ horizon [17, 67]: the medium screens the infrared part of the parton cloud surrounding the quark, thus reducing the dressed heavy quark mass. It is essential that the quark is heavy not due to a large bare quark mass, but due to quantum dressing by the gauge field fluctuations: this allows the thermal medium to eliminate the soft IR fluctuations and make the quark mass smaller in the medium.

Now, if we think of the shock wave considered here as of a boosted thermal medium (which is one of the possibilities), then the time scale of the thermal motion of the medium particles would be Lorentz-dilated in the rest frame of the quark: $\tau_{t h} \sim(1 / T) \gamma$. As $\gamma \gg 1$, the dilated thermal time scale $\tau_{t h}$ is much longer than any other time scale in the problem. This is a well-known feature of the parton model in the infinite momentum frame, in which the partons appear "frozen in time" to the probe interacting with them. Hence our static heavy quark does not "feel" the thermal motion of the particles in the shock wave over the typical interaction time scale between the quark and a shock wave particle. This is why the heavy quark mass does not get modified by the shock wave, as we saw in Eq. (30): the lack of thermal motion on the interaction time scale means no screening for the infrared partons in the quark wave function. Indeed there is an IR screening due to saturation effects in the shock [60, 66], which may lead to quark mass modification if we integrate over $z$ in Eq. (30) only up to the horizon of the induced metric on the string, as was done in $[66,67]$. Our qualitative picture also explains why the thermal effects are not relevant for the quark energy loss: in the infinite momentum frame discussed here the thermal motion is irrelevant, but the quark still experiences a drag force. We conclude that the drag force indeed does not depend on the thermal motion, and is thus independent of whether the medium is thermal or not. This is exactly the same conclusion as we obtained by explicit derivation above.

\section{Acknowledgments}

We are grateful to Alberto Guijosa, Miklos Gyulassy, Ulrich Heinz, Boris Kopeliovich, Hong Liu, Cyrille Marquet, Samir Mathur, Jorge Noronha, Krishna Rajagopal, Anastasios Taliotis, Derek Teaney, and Bill Zajc for fruitful discussions.

This work is supported by the Office of Nuclear Physics, of the Office of Science, of the U.S. Department of Energy under Grant No. DE-FG02-05ER41377.

\section{A Other Solutions to the EOM}

The argument used to derive the stable static solutions $x(t, z)=x_{0}, x_{0} \pm \sqrt{\mu} z^{3} / 3$ required the string to hang from a $z$ smaller than both $\sqrt[4]{\mu}$ and $\sqrt{C}$ down to the stack of D3 branes at $z=\infty$ so that, in order for the solution to remain real, both the numerator and denominator 
of the radical in Eq. (12) had to change sign at precisely the same value of $z$. However strings with a turning point may also satisfy this reality condition.

For such a solution $x(z)$ to exist, $z(x)$ should increase with $x$ until the turning point, where the slope of $z(x)$ must be 0 (note the use of the inverse function). If we denote the value of $z$ for which the numerator of the radical in Eq. (12) is zero as $z_{c}$ and the value of $z$ for which the denominator is zero as $z_{\max }$, then

$$
\begin{array}{cc}
1-\mu z_{c}^{4}=0 & 1-C^{2} z_{\max }^{4}=0 \\
\Rightarrow \quad z_{c}=\mu^{-1 / 4} & z_{\max }=C^{-1 / 2}
\end{array}
$$

The condition for turning, $d z / d x=0$, thus requires the string solution to extend up to $z=z_{\max }$.

Concentrating on stable string solutions, ones for which the string configuration is purely real, we can consider two cases: (1) $z_{M}<z_{\max }<z_{c}$ and (2) $z_{M}>z_{\max }$, $z_{c}$. The latter case may be solved analytically in closed form for the two shape possibilities: (a) string endpoints at plus and minus infinity in the $x^{3}$ direction, both terminating on the D7 or the stack of D3 branes with a hump at $x_{0}$ that passes through $z_{\max }$ and (b) a hanging string solution from the D7 brane to the D3 branes. There is considerable freedom in these solutions as $C$ is no longer fixed. However none are physical due to the speed limit condition, Eq. (27).

Unlike in the black hole metric one may also solve the hanging "meson" case (1) analytically. For simplicity let us consider infinitely massive quarks with $z_{M}=0$. The parameterization from $x=-r / 2$ to 0 for a string with ends at $(x, z)=( \pm r / 2,0)$ may be found by direct integration of the first integral of the motion, Eq. (12):

$$
\begin{aligned}
\xi(z) & =-\frac{r}{2}+\frac{1}{z_{\max }^{2}} \int_{0}^{z} d z^{\prime} z^{\prime 2} \sqrt{\frac{1-\mu z^{\prime 4}}{1-\frac{z^{\prime 4}}{z_{\max }^{4}}}} \\
& =-\frac{r}{2}+\frac{z^{3}}{3 z_{\max }^{2}} \mathrm{~F}_{1}\left(\frac{3}{4} ;-\frac{1}{2}, \frac{1}{2} ; \frac{7}{4} ; \mu z^{4}, \frac{z^{4}}{z_{\max }^{4}}\right),
\end{aligned}
$$

where $\mathrm{F}_{1}$ is Appell's hypergeometric function of the first kind (to construct the solution in the interval from $x=0$ to $r / 2$ simply reflect Eq. (A.2) across $x=0$ ); this solution is shown in Fig. 4. The solution in Eq. (A.2) complements the results of [60] in which the string profile was found for a dipole oriented perpendicular to the direction of shock wave motion, by providing the solution for a dipole oriented parallel to the shock velocity.

Imposing the turning condition on Eq. (A.2), i.e., that in the middle of the string at $x=0$ one has the turning point $z=z_{\max }$, yields the following condition for $z_{\max }$ :

$$
\frac{r}{2}=\frac{\sqrt{\pi} \Gamma(7 / 4)}{3 \Gamma(5 / 4)} z_{\max } \mathrm{F}\left(\frac{3}{4},-\frac{1}{2} ; \frac{5}{4} ; \mu z_{\max }^{4}\right) .
$$

Numerical analysis shows that for $r>r_{\text {crit }} \approx 0.85 z_{c}=0.85 \mu^{-1 / 4}$ the solution for $z_{\text {max }}$ becomes complex-valued.

For $r<r_{\text {crit }}$ the singlet configuration for the quark anti-quark pair, Eq. (A.2), suffers no energy loss: the momentum lost by the leading heavy quark is exactly canceled by the momentum gain of the heavy quark that follows behind. However, for separations $r>r_{\text {crit }}$ the system is unstable to decay into a color-adjoint state, possibly leading to complete dissolution of the meson. To completely answer this question a full study of the roots for $z_{\max }$ in the case of a general orientation of the dipole with respect to the shock velocity similar to what was done in [68] needs to be carried out: such analysis goes beyond the scope of this work. 


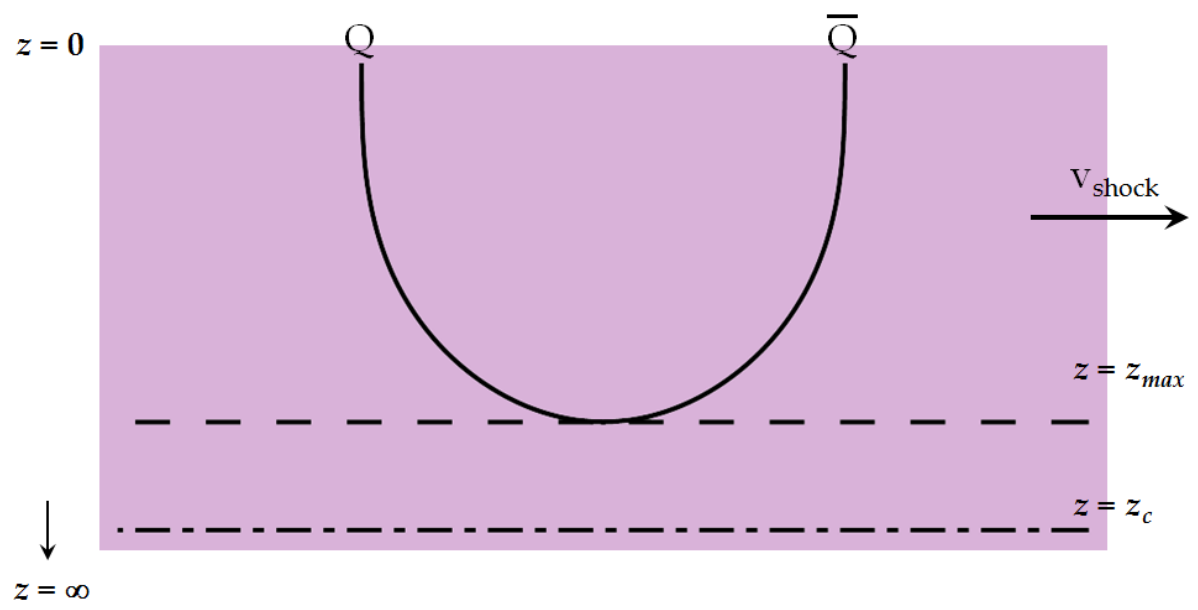

Figure 4: Visualization of the hanging meson configuration, Eq. (A.2). Note that $z_{c}>z_{\max }$, and the string turns precisely at $z=z_{\max }$.

\section{References}

[1] J. M. Maldacena, The large $N$ limit of superconformal field theories and supergravity, Adv. Theor. Math. Phys. 2, 231 (1998) [arXiv:hep-th/9711200].

[2] S. S. Gubser, I. R. Klebanov, and A. M. Polyakov, Gauge theory correlators from noncritical string theory, Phys. Lett. B428, 105 (1998) [arXiv:hep-th/9802109].

[3] E. Witten, Anti-de Sitter space and holography, Adv. Theor. Math. Phys. 2, 253 (1998) [arXiv:hep-th/9802150].

[4] O. Aharony, S. S. Gubser, J. M. Maldacena, H. Ooguri, and Y. Oz, Large $N$ field theories, string theory and gravity, Phys. Rept. 323, 183 (2000) [arXiv:hep-th/9905111].

[5] S. S. Gubser and A. Karch, From gauge-string duality to strong interactions: a Pedestrian's Guide (2009) [arXiv:0901.0935].

[6] S. S. Gubser, S. S. Pufu, F. D. Rocha, and A. Yarom, Energy loss in a strongly coupled thermal medium and the gauge-string duality (2009) [arXiv:0902.4041].

[7] S. Wicks, W. Horowitz, M. Djordjevic, and M. Gyulassy, Elastic, Inelastic, and Path Length Fluctuations in Jet Tomography, Nucl. Phys. A784, 426 (2007) [arXiv:nucl-th/ 0512076].

[8] P. Kovtun, D. T. Son, and A. O. Starinets, Viscosity in strongly interacting quantum field theories from black hole physics, Phys. Rev. Lett. 94, 111601 (2005) [arXiv:hep-th/ 0405231].

[9] R. A. Janik and R. B. Peschanski, Asymptotic perfect fluid dynamics as a consequence of AdS/CFT, Phys. Rev. D73, 045013 (2006) [arXiv:hep-th/0512162].

[10] H. Liu, K. Rajagopal, and U. A. Wiedemann, An AdS/CFT calculation of screening in a hot wind, Phys. Rev. Lett. 98, 182301 (2007) [arXiv:hep-ph/0607062]. 
[11] C. P. Herzog, Energy loss of heavy quarks from asymptotically AdS geometries, JHEP 09, 032 (2006) [arXiv:hep-th/0605191].

[12] S. S. Gubser, Drag force in AdS/CFT, Phys. Rev. D74, 126005 (2006) [arXiv:hep-th/ 0605182].

[13] J. Casalderrey-Solana and D. Teaney, Transverse momentum broadening of a fast quark in a $N=4$ Yang Mills plasma, JHEP 04, 039 (2007) [arXiv:hep-th/0701123].

[14] W. A. Horowitz and M. Gyulassy, Heavy quark jet tomography of $\mathrm{Pb}+\mathrm{Pb}$ at $\mathrm{LHC}$ : AdS/CFT drag or pQCD energy loss?, Phys. Lett. B666, 320 (2008) [arXiv:0706.2336].

[15] A. Mikhailov, Nonlinear waves in AdS/CFT correspondence (2003) [arXiv:hep-th/ 0305196].

[16] S.-J. Sin and I. Zahed, Holography of radiation and jet quenching, Phys. Lett. B608, 265 (2005) [arXiv:hep-th/0407215].

[17] M. Chernicoff and A. Guijosa, Acceleration, Energy Loss and Screening in Strongly-Coupled Gauge Theories, JHEP 06, 005 (2008) [arXiv:0803.3070].

[18] D. E. Kharzeev, Universal upper bound on the energy of a parton escaping from the strongly coupled quark-gluon matter (2008) [arXiv:0806.0358].

[19] P. M. Chesler and L. G. Yaffe, The stress-energy tensor of a quark moving through a strongly-coupled $N=4$ supersymmetric Yang-Mills plasma: comparing hydrodynamics and AdS/CFT, Phys. Rev. D78, 045013 (2008) [arXiv:0712.0050].

[20] F. Karsch, E. Laermann, and A. Peikert, The pressure in 2, 2+1 and 3 flavour QCD, Phys. Lett. B478, 447 (2000) [arXiv:hep-lat/0002003].

[21] M. Cheng et al., The QCD Equation of State with almost Physical Quark Masses, Phys. Rev. D77, 014511 (2008) [arXiv:0710.0354].

[22] S. S. Gubser, I. R. Klebanov, and A. W. Peet, Entropy and Temperature of Black 3-Branes, Phys. Rev. D54, 3915 (1996) [arXiv:hep-th/9602135].

[23] I. R. Klebanov, TASI lectures: Introduction to the AdS/CFT correspondence (2000) [arXiv:hep-th/0009139].

[24] D. Teaney and E. V. Shuryak, An unusual space-time evolution for heavy ion collisions at high energies due to the QCD phase transition, Phys. Rev. Lett. 83, 4951 (1999) [arXiv:nucl-th/9904006].

[25] P. F. Kolb, J. Sollfrank, and U. W. Heinz, Anisotropic transverse flow and the quark-hadron phase transition, Phys. Rev. C62, 054909 (2000) [arXiv:hep-ph/0006129].

[26] P. Huovinen, P. F. Kolb, U. W. Heinz, P. V. Ruuskanen, and S. A. Voloshin, Radial and elliptic flow at RHIC: further predictions, Phys. Lett. B503, 58 (2001) [arXiv:hep-ph/ 0101136]. 
[27] D. Teaney, Effect of shear viscosity on spectra, elliptic flow, and Hanbury Brown-Twiss radii, Phys. Rev. C68, 034913 (2003) [arXiv:nucl-th/0301099].

[28] H. Song and U. W. Heinz, Causal viscous hydrodynamics in 2+1 dimensions for relativistic heavy-ion collisions, Phys. Rev. C77, 064901 (2008) [arXiv:0712.3715].

[29] S. S. Adler et al. (PHENIX), Nuclear modification of electron spectra and implications for heavy quark energy loss in $A u+A u$ collisions at $s(N N)^{* *}(1 / 2)=200-G e V$, Phys. Rev. Lett. 96, 032301 (2006) [arXiv:nucl-ex/0510047].

[30] B. I. Abelev et al. (STAR), Transverse momentum and centrality dependence of high-pt non-photonic electron suppression in Au+Au collisions at $\sqrt{s_{N N}}=200 \mathrm{GeV}$, Phys. Rev. Lett. 98, 192301 (2007) [arXiv:nucl-ex/0607012].

[31] A. Adare et al. (PHENIX), Energy Loss and Flow of Heavy Quarks in Au+Au Collisions at $\sqrt{s_{N} N}=200 \mathrm{GeV}$, Phys. Rev. Lett. 98, 172301 (2007) [arXiv:nucl-ex/0611018].

[32] A. Mischke (STAR), Heavy-flavor particle correlations in STAR via electron azimuthal correlations with $D^{0}$ mesons, J. Phys. G35, 104117 (2008) [arXiv:0804.4601].

[33] Y. Morino (PHENIX), Measurement of charm and bottom production in $p+p$ collisions at $\sqrt{s}=200 \mathrm{GeV}$ at RHIC-PHENIX, J. Phys. G35, 104116 (2008) [arXiv:0805.3871].

[34] J. Adams et al. (STAR), Distributions of charged hadrons associated with high transverse momentum particles in $p$ and $A u+A u$ collisions at $s(N N)^{* *}(1 / 2)=200-G e V$, Phys. Rev. Lett. 95, 152301 (2005) [arXiv:nucl-ex/0501016].

[35] S. S. Adler et al. (PHENIX), Modifications to di-jet hadron pair correlations in $A u+A u$ collisions at $s(N N)^{* *}(1 / 2)=200-G e V$, Phys. Rev. Lett. 97, 052301 (2006) [arXiv:nucl-ex/ 0507004].

[36] A. Adare et al. (PHENIX), Dihadron azimuthal correlations in Au+Au collisions at $\sqrt{s_{N N}}=200 \mathrm{GeV}$, Phys. Rev. C78, 014901 (2008) [arXiv:0801.4545].

[37] J. G. Ulery (STAR), Two- and three-particle jet correlations from STAR, Nucl. Phys. A774, 581 (2006) [arXiv:nucl-ex/0510055].

[38] C. Zhang (PHENIX), Studying the medium modification of jets via high-p(T) hadron angular correlations, J. Phys. G34, S671 (2007).

[39] H. Stoecker, Collective Flow signals the Quark Gluon Plasma, Nucl. Phys. A750, 121 (2005) [arXiv:nucl-th/0406018].

[40] J. Casalderrey-Solana, E. V. Shuryak, and D. Teaney, Conical flow induced by quenched QCD jets, J. Phys. Conf. Ser. 27, 22 (2005) [arXiv:hep-ph/0411315].

[41] J. J. Friess, S. S. Gubser, G. Michalogiorgakis, and S. S. Pufu, The stress tensor of a quark moving through $N=4$ thermal plasma, Phys. Rev. D75, 106003 (2007) [arXiv:hep-th/ 0607022]. 
[42] B. Betz, M. Gyulassy, J. Noronha, and G. Torrieri, Anomalous Conical Di-jet Correlations in $p Q C D$ vs AdS/CFT (2008) [arXiv:0807.4526].

[43] R. Baier, Y. L. Dokshitzer, A. H. Mueller, S. Peigne, and D. Schiff, Radiative energy loss of high energy quarks and gluons in a finite-volume quark-gluon plasma, Nucl. Phys. B483, 291 (1997) [arXiv:hep-ph/9607355].

[44] M. Gyulassy, P. Levai, and I. Vitev, Reaction operator approach to non-Abelian energy loss, Nucl. Phys. B594, 371 (2001) [arXiv:nucl-th/0006010].

[45] I. Vitev and M. Gyulassy, High $p_{T}$ tomography of $d+A u$ and $A u+A u$ at SPS, RHIC, and LHC, Phys. Rev. Lett. 89, 252301 (2002) [arXiv:hep-ph/0209161].

[46] A. Adare et al. (PHENIX), Suppression pattern of neutral pions at high transverse momentum in $A u+A u$ collisions at $\sqrt{s_{N} N}=200 \mathrm{GeV}$ and constraints on medium transport coefficients, Phys. Rev. Lett. 101, 232301 (2008) [arXiv:0801.4020].

[47] G. Lin (STAR), First STAR results on piO production over an extended $p(T)$-range from 200-GeV Au + Au collisions, J. Phys. G35, 104046 (2008).

[48] S. S. Adler et al. (PHENIX), High transverse momentum $\eta$ meson production in $p^{+} p$, $d^{+} A u$ and $A u+A u$ collisions at $S(N N)^{(1 / 2)}=200-G e V$, Phys. Rev. C75, 024909 (2007) [arXiv:nucl-ex/0611006].

[49] S. S. Adler et al. (PHENIX), Centrality dependence of direct photon production in $s(N N)^{* *}(1 / 2)=200-G e V A u+A u$ collisions, Phys. Rev. Lett. 94, 232301 (2005) [arXiv:nucl-ex/0503003].

[50] K. Miki (PHENIX), High-p(T) direct photon spectra and azimuthal anisotropy measurements in 200-GeV Au + Au collisions at RHIC-PHENIX, J. Phys. G35, 104122 (2008).

[51] M. Djordjevic, M. Gyulassy, R. Vogt, and S. Wicks, Influence of bottom quark jet quenching on single electron tomography of $A u+A u$, Phys. Lett. B632, 81 (2006) [arXiv:nucl-th/ 0507019].

[52] N. Armesto, M. Cacciari, A. Dainese, C. A. Salgado, and U. A. Wiedemann, How sensitive are high-p (T) electron spectra at RHIC to heavy quark energy loss?, Phys. Lett. B637, 362 (2006) [arXiv:hep-ph/0511257].

[53] S. S. Adler et al. (PHENIX), A detailed study of high-p(T) neutral pion suppression and azimuthal anisotropy in $A u+$ Au collisions at $s(N N)^{* *}(1 / 2)=200-G e V$, Phys. Rev. C76, 034904 (2007) [arXiv:nucl-ex/0611007].

[54] B. I. Abelev et al. (STAR), Centrality dependence of charged hadron and strange hadron elliptic flow from $\sqrt{s_{N N}}=200 \mathrm{GeV}$ Au+Au collisions, Phys. Rev. C77, 054901 (2008) [arXiv:0801.3466].

[55] M. Gyulassy and X.-n. Wang, Multiple collisions and induced gluon Bremsstrahlung in QCD, Nucl. Phys. B420, 583 (1994) [arXiv:nucl-th/9306003]. 
[56] J. C. Collins, Hard scattering in QCD with polarized beams, Nucl. Phys. B394, 169 (1993) [arXiv:hep-ph/9207265].

[57] J. Collins and J.-W. Qiu, $k_{T}$ factorization is violated in production of high-transversemomentum particles in hadron-hadron collisions, Phys. Rev. D75, 114014 (2007) [arXiv:0705.2141].

[58] S. de Haro, S. N. Solodukhin, and K. Skenderis, Holographic reconstruction of spacetime and renormalization in the AdS/CFT correspondence, Commun. Math. Phys. 217, 595 (2001) [arXiv:hep-th/0002230].

[59] C. Fefferman and C. R. Graham, Conformal Invariants, in Élie Cartan et les Mathématiques d'aujourd'hui, 95-116 (Astérisque, 1985).

[60] J. L. Albacete, Y. V. Kovchegov, and A. Taliotis, DIS on a Large Nucleus in AdS/CFT, JHEP 07, 074 (2008) [arXiv:0806.1484].

[61] J. L. Albacete, Y. V. Kovchegov, and A. Taliotis, Modeling Heavy Ion Collisions in AdS/CFT, JHEP 07, 100 (2008) [arXiv:0805.2927].

[62] A. Karch and E. Katz, Adding flavor to AdS/CFT, JHEP 06, 043 (2002) [arXiv:hep-th/ 0205236].

[63] C. P. Herzog, A. Karch, P. Kovtun, C. Kozcaz, and L. G. Yaffe, Energy loss of a heavy quark moving through $N=4$ supersymmetric Yang-Mills plasma, JHEP 07, 013 (2006) [arXiv:hep-th/0605158].

[64] C. Bachas, D-brane dynamics, Phys. Lett. B374, 37 (1996) [arXiv:hep-th/9511043].

[65] S. S. Gubser, Momentum fluctuations of heavy quarks in the gauge-string duality, Nucl. Phys. B790, 175 (2008) [arXiv:hep-th/0612143].

[66] F. Dominguez, C. Marquet, A. H. Mueller, B. Wu, and B.-W. Xiao, Comparing energy loss and $p_{\perp}$-broadening in perturbative $Q C D$ with strong coupling $\mathcal{N}=4 S Y M$ theory, Nucl. Phys. A811, 197 (2008) [arXiv:0803.3234].

[67] G. Beuf, C. Marquet, and B.-W. Xiao, Heavy-quark energy loss and thermalization in a strongly coupled SYM plasma (2008) [arXiv:0812.1051].

[68] H. Liu, K. Rajagopal, and U. A. Wiedemann, Wilson loops in heavy ion collisions and their calculation in AdS/CFT, JHEP 03, 066 (2007) [arXiv:hep-ph/0612168]. 